\title{
Alemtuzumab: a review of efficacy and risks in the treatment of relapsing remitting multiple sclerosis
}

\author{
This article was published in the following Dove Press journal: \\ Therapeutics and Clinical Risk Management \\ 14 July 2017 \\ Number of times this article has been viewed
}

\section{Cristina Guarnera \\ Placido Bramanti \\ Emanuela Mazzon \\ Experimental Neurology Laboratory, IRCCS Centro Neurolesi "Bonino- Pulejo", Via Provinciale Palermo, Contrada Casazza, Messina, Italy}

\begin{abstract}
Alemtuzumab is a selective humanized monoclonal antibody directed against the CD52 antigen, and has been found to be a powerful treatment for relapsing remitting multiple sclerosis. Alemtuzumab demonstrated high efficacy in several clinical studies. The risk of relapse and sustained accumulation of disability showed significant reduction in the Phase II CAMMS223 and the Phase III clinical trials CARE MS I and CARE MS II. The data presented at the 32nd Congress of the European Committee for Treatment and Research in Multiple Sclerosis confirmed these results. After completion of a 1-year treatment cycle, alemtuzumab showed a sustained effect. Although the efficacy of alemtuzumab has been widely proven, several severe adverse effects have been reported with its use. Infusion-associated reactions, increased risk of infections, and secondary autoimmunity have been associated with alemtuzumab. Autoimmune disease - mainly of the thyroid - has been reported. Immune thrombocytopenic purpura and autoimmune nephropathies have been observed less frequently. These adverse effects, given the short period of alemtuzumab marketing for relapsing remitting multiple sclerosis, require strict monitoring.
\end{abstract}

Keywords: effectiveness, clinical studies, follow-up, adverse events

\section{Introduction}

Alemtuzumab is a humanized monoclonal antibody selectively directed against the CD52 antigen on T- and B-lymphocytes. ${ }^{1}$ Treatment with alemtuzumab produces a depletion of circulating B- and T-lymphocytes, resulting in a decrease of relapses in relapsing remitting multiple sclerosis (RRMS). Alemtuzumab is administered by infusion in 2 treatment cycles. The first treatment cycle includes 1 alemtuzumab $12 \mathrm{mg} / \mathrm{d}$ infusion for 5 consecutive days. After 12 months from the first infusion cycle, the second cycle is administered. The second cycle consists of 1 infusion per day for 3 consecutive days. The European Medicines Agency (EMA) and the US Food and Drug Administration (FDA) approved marketing authorization of alemtuzumab for the treatment of adult patients with RRMS. ${ }^{1,2}$ Alemtuzumab is currently authorized in $>40$ countries. We review the key findings of the efficacy of alemtuzumab in clinical trials, together with recently published studies, providing an overview of its adverse effects.

\section{Materials and methods}

In this review, we make reference to data obtained from the PubMed database. To evaluate the efficacy and safety of alemtuzumab in RRMS, randomized clinical trials, their extensions and follow-up studies are analyzed. For a more comprehensive assessment of the drug, cohort studies and case reports have been taken into 
consideration. The end points of efficacy were the relapse rate and the sustained accumulation of disability (SAD). The end points of safety were the adverse effects reported with alemtuzumab treatment. Furthermore, in the manuscript, we have included data concerning safety monitoring programs found on the US FDA and the EMA websites.

\section{Efficacy outcomes}

Alemtuzumab has been evaluated in different clinical studies. In initial studies with secondary progressive multiple sclerosis, it did not show improvements, signaling a therapeutic opportunity in early form of disease. ${ }^{3-6}$ The Phase $\mathrm{II}^{7}$ and Phase III $^{8,9}$ clinical studies assessed its efficacy in patients with RRMS. In these studies, alemtuzumab demonstrated greater efficacy in several clinical outcomes compared to active interferon (IFN) $\beta$ 1a. The randomized Phase II trial CAMMS2237 ${ }^{7}$ compared intravenous alemtuzumab with subcutaneous (SC) IFN $\beta$ 1a over a 36-month period in naïve patients. Alemtuzumab 12 and $24 \mathrm{mg} / \mathrm{d}$ was administered for 5 days in the first month, for 3 days in the twelfth month and after 24 months for 3 days (at the discretion of the physician). IFN $\beta$ 1a $44 \mu \mathrm{g}$ was given 3 times a week. The study included patients with early active disease. Active disease was defined by $\geq 2$ relapses in the previous 2 years and $\geq 1$ gadolinium (Gd)-positive lesion, Expanded Disability Status Scale (EDSS) score $\leq 3$ and onset $\leq 3$ years (Table 1 ). The results of this study showed a $74 \%$ reduction in the risk of relapse, a $71 \%$ reduction in the risk of 6 -month $\operatorname{SAD}(P<0.001)$ and an improvement of 0.39 points in EDSS scores with alemtuzumab treatment. The annualized relapse rate (ARR) was 0.10 with alemtuzumab versus 0.36 with IFN $\beta 1 \mathrm{a}^{7}$ Subsequently, the Phase III trial CARE MS I ${ }^{8}$ confirmed most results of the previous study. The 2-year randomized controlled study compared alemtuzumab $12 \mathrm{mg}$ (once per day for 5 days at month 1 and for 3 days at month 12) with SC IFN $\beta$ 1a $44 \mu \mathrm{g}$ (3 times weekly) in previously untreated patients with active disease. ${ }^{8}$ In the study, the criteria of active disease were $\geq 2$ relapses in the previous 2 years and $\geq 1$ relapse in the previous year, EDSS score $\leq 3$ and onset $\leq 5$ years (Table 1). With a risk reduction of $54.9 \%$ and ARR 0.18 versus 0.39 of IFN $\beta$ 1a, alemtuzumab confirmed its superior effect on clinical relapse. ${ }^{8}$ However, significant results in SAD (6-month SAD 8\% with alemtuzumab versus $11 \%$ with IFN $\beta$ 1a) were not observed. CARE MS II ${ }^{9}$ was the randomized controlled Phase III study of alemtuzumab versus IFN $\beta$ 1a in patients with active disease and inadequate response (at least 1 relapse) to previous therapy with IFN $\beta$ or glatiramer acetate (GA). In this trial, active disease was clinically defined by $\geq 2$ relapses in the previous 2 years and $\geq 1$ relapse in the previous year, EDSS score $\leq 5$ and onset $\leq 10$ years. The study confirmed the results of CAMMS223 ${ }^{7}$ in terms of all clinical outcomes. Higher efficacy compared to IFN $\beta$ 1a was confirmed regardless of the previous disease-modifying therapies (DMTs). ${ }^{9}$ The risk reduction for relapse rate was $49.4 \%$. ARR was 0.26 with alemtuzumab versus 0.52 with IFN $\beta$ 1a. Unlike CARE MS I, ${ }^{8}$ in this study, alemtuzumab

Table I Outcomes of Phase II and Phase III clinical trials of alemtuzumab in RRMS

\begin{tabular}{|c|c|c|c|}
\hline \multirow[t]{2}{*}{ Features } & \multirow{2}{*}{$\frac{\text { CAMMS223 }^{7}}{12 \text { and } 24 \mathrm{mg}}$} & \multirow{2}{*}{$\begin{array}{l}\text { CARE MS I } \\
2 \mathrm{mg}\end{array}$} & \multirow{2}{*}{$\begin{array}{l}\text { CARE MS II } \\
2 \mathrm{mg}\end{array}$} \\
\hline & & & \\
\hline Inclusion criteria & $\begin{array}{l}\text { Active RRMS: } \geq 2 \text { relapses in } \\
\text { the previous } 2 \text { years and } \geq 1 \\
\text { Gd-positive lesion; treatment naïve; } \\
\text { EDSS } \leq 3 \text {; onset } \leq 3 \text { years }\end{array}$ & $\begin{array}{l}\text { Active RRMS: } \geq 2 \text { relapses in the } \\
\text { previous } 2 \text { years and } \geq 1 \text { relapse } \\
\text { in the previous year; treatment } \\
\text { naïve; EDSS } \leq 3 \text {; onset } \leq 5 \text { years }\end{array}$ & $\begin{array}{l}\text { Active RRMS: } \geq 2 \text { relapses in the } \\
\text { previous } 2 \text { years and } \geq 1 \text { relapse in } \\
\text { the previous year; relapsing on prior } \\
\text { DMT; EDSS } \leq 5 \text {; onset } \leq 10 \text { years }\end{array}$ \\
\hline $\begin{array}{l}\text { Relapse rate reduction with } \\
\text { alemtuzumab compared to } \\
\text { that with IFN } \beta \text { Ia }\end{array}$ & $74 \%(P<0.001)$ & $54.9 \%(P<0.000 I)$ & $49.4 \%(P<0.0001)$ \\
\hline ARR & 0.10 versus 0.36 & 0.18 versus 0.39 & 0.26 versus 0.52 \\
\hline $\begin{array}{l}\text { Reduction in SAD of } \\
\text { alemtuzumab compared } \\
\text { to IFN } \beta \text { Ia }\end{array}$ & $\begin{array}{l}71 \% \text { reduction: } 75 \% \text { with } \\
\text { alemtuzumab } 12 \mathrm{mg} \text { and } 67 \% \\
\text { with alemtuzumab } 24 \mathrm{mg}\end{array}$ & No significant reduction & $42 \%$ reduction \\
\hline Mean EDSS change & $\begin{array}{l}0.39 \text { point improvement with } \\
\text { alemtuzumab; worsening by } \\
0.38 \text { points with IFN } \beta \text { Ia }(P<0.00 \text { I) }\end{array}$ & No changes & $\begin{array}{l}0.17 \text { point improvement with } \\
\text { alemtuzumab; worsening by } \\
0.24 \text { points with IFN } \beta \text { Ia }(P<0.000 \text { I })\end{array}$ \\
\hline Patients relapse free & $\begin{array}{l}80 \% \text { with alemtuzumab: } 77 \% \text { with } \\
12 \mathrm{mg} \text { dose, } 84 \% \text { with } 24 \mathrm{mg} \text { dose; } \\
52 \% \text { with IFN } \beta \text { Ia; } P<0.00 \text { I } \\
\text { (at } 36 \text { months) }\end{array}$ & $\begin{array}{l}78 \% \text { with alemtuzumab; } 59 \% \\
\text { with IFN } \beta \text { Ia; } P<0.000 \text { I } \\
\text { (at } 2 \text { years) }\end{array}$ & $\begin{array}{l}65 \% \text { with alemtuzumab; } 47 \% \text { with } \\
\text { IFN } \beta \text { Ia; } P<0.000 \text { I (at } 2 \text { years) }\end{array}$ \\
\hline
\end{tabular}

Abbreviations: ARR, annual relapse rate; DMT, disease-modifying therapy; EDSS, Expanded Disability Status Scale; Gd, gadolinium; IFN $\beta$ Ia, interferon $\beta$ Ia; RRMS, relapsing remitting multiple sclerosis; SAD, sustained accumulation of disability. 
showed a significant reduction in disability progression compared to IFN $\beta$ 1a. The risk reduction for SAD was $42 \%$ (12.7\% with alemtuzumab versus $21.1 \%$ with IFN $\beta$ 1a). Alemtuzumab also demonstrated higher improvement rate in mean EDSS score ( 0.17 points) compared to IFN $\beta 1 \mathrm{a} .{ }^{9} \mathrm{~A}$ post hoc analysis of CARE MS II $^{9}$ further reinforced the efficacy of alemtuzumab in disability improvement compared to IFN $\beta$ 1a. This analysis showed improvement in the Multiple Sclerosis Functional Composite (MFSC) score, mostly on arm function [9-Hole Peg Test (9-HPT)], and in the Sloan low contrast letter acuity (SLCLA) score. In addition, about half of the patients treated with alemtuzumab had an improvement in EDSS scores $(P<0.0001)$ in all the 7 EDSS functional domains. ${ }^{10}$ Moreover, data from follow-up and extension studies of patients from Phase II and Phase III trials reproduced results previously obtained on relapse rate and disability. In a follow-up study, ${ }^{11}$ over an average period of 7 years, alemtuzumab produced stabilization or improvement of disability in $67.8 \%$ of the patients with early active disease (established by 6-month SAD). In this cohort study, $52 \%$ of patients required 2 cycles of alemtuzumab over a 7-year period. ${ }^{11}$ In the 5-year extension study of CAMMS223, ${ }^{12}$ alemtuzumab showed a $69 \%$ reduction $(P<0.0001)$ in relapse rate and a $72 \%$ reduction $(P<0.0001)$ in SAD. ARR was 0.11 with alemtuzumab versus 0.35 with IFN $\beta$ 1a. ${ }^{12}$ Four-year data of CARE MS extension studies showed that after alemtuzumab treatment, more than half of the patients had no evidence of disease activity (NEDA). In the follow-up of the CARE MS II study, ${ }^{13,14}$ this result increased (70\%). Moreover, alemtuzumab showed significant improvement of disability in $41 \%$ of patients with 6 months of sustained reduction in disability (SRD) and in 30\% of patients with 12-month SRD (determined by a reduction in EDSS scores). ${ }^{13}$ All these results demonstrated the clinical efficacy of alemtuzumab in RRMS. Both in naïve patients, as induction therapy, and in patients with inadequate response to previous therapy, as escalation therapy, its efficacy in active disease has been demonstrated. The extension study, recently presented at the 32 nd Congress of the European Committee for Treatment and Research in Multiple Sclerosis (ECTRIMS), showed that after alemtuzumab treatment, more than half of the patients from the CARE $\mathrm{MS}^{8,9}$ trials maintained positive effects over a 6-year period. ${ }^{15}$ More than $90 \%$ of patients from CARE MS studies were enrolled in this extension study. Criteria used for retreatment of alemtuzumab were at least 1 relapse or 2 new or enlarging (brain or spinal) lesions. An additional alemtuzumab treatment in about more than half of patients from pivotal studies (64\% from CARE MS I ${ }^{8}$ and 55\% CARE MS II, ${ }^{9}$ respectively) was not required. ARR was low over a 6-year period (0.12 and 0.15 at the sixth year), as seen in pivotal studies. Moreover, many of the patients from CARE $\mathrm{MS}^{8,9}$ studies ( $77 \%$ and $72 \%$, respectively) maintained the same level of disability. The study recorded an improvement in EDSS scores in $34 \%$ and $43 \%$, respectively, of patients with disability prior to treatment with alemtuzumab. Most patients had NEDA on MRI and less brain volume loss compared with that observed in the pivotal trials (specifically, median annual brain volume loss in extension was $\leq-0.20$; in CARE MS I, it was $-0.59 \%$ at year 1 and $-0.25 \%$ at year 2 ; and in CARE MS II, it was $-0.48 \%$ at year 1 and $-0.22 \%$ at year 2$).{ }^{15}$ The main result of this study was a sustained effect of alemtuzumab after a completed 1-year treatment cycle. Finally, in a recent international study on behalf of the MSBase Study Group (published in February 2017), alemtuzumab proved superior to IFN $\beta$ and fingolimod in reducing relapse rate, demonstrating similar effect to that of natalizumab. ${ }^{17}$ This cohort study compared alemtuzumab with 3 other agents, namely, natalizumab, fingolimod and IFN $\beta$ 1a, in patients with RRMS treated for up to 5 years. In the study, the following dose schedules were used: intravenous alemtuzumab 12 and $24 \mathrm{mg}$ once per day for 5 days at the first cycle and for 3 days at the second cycle; SC IFN $\beta$ 1a $44 \mu \mathrm{g} 3$ times weekly; oral fingolimod $0.5 \mathrm{mg}$ once a day; and intravenous natalizumab $300 \mu \mathrm{g}$ every 4 weeks. The study enrolled patients with EDSS score up to 6.0 and first symptom of disease not $>10$ years earlier. In the study, alemtuzumab showed a lower ARR compared to IFN $\beta$ 1a over 5 years after the first treatment $(0.19$ versus $0.53, P<0.0001)$ and fingolimod over 3 years $(0.15$ versus $0.34, P<0.0001)$. Instead, ARR was similar to natalizumab over 4 years $(0.20$ versus 0.19 , $P=0.78) .{ }^{16}$ Regarding the disability accumulation, alemtuzumab showed similar probability of disability accumulation compared to the 3 drugs, with no differences in the cumulative hazard of 6-month confirmed disability accumulation (alemtuzumab versus IFN $\beta$ 1a, cumulative hazard of disability accumulation $0.66, P=0.37$; alemtuzumab versus fingolimod $1.27, P=0.67$; natalizumab $0.81, P=0.60)$. Regarding the disability improvement, no differences in the cumulative probability of 6-month confirmed disability improvement compared to IFN $\beta$ 1a (hazard ratio $0.98, P=0.93$ ) and fingolimod (hazard ratio $0.50, P=0.18$ ) were seen. However, the drug showed lower probability of disability improvement than natalizumab, having a lower cumulative hazard of disability improvement (hazard ratio 0.35, $P=0.0006$ ) than natalizumab. Natalizumab therefore showed a slightly higher disability improvement rate compared to alemtuzumab in the study, 
especially during the first years of therapy. Since the disability progression over the 4-year period was similar, the efficacy of the 2 agents was similar. These data have been confirmed by a subsequent analysis over a 12 -month interval. ${ }^{17}$ In this study, comparison of alemtuzumab with IFN $\beta$ 1a also substantiated the findings of pivotal trials of alemtuzumab.

\section{Adverse events}

In the clinical studies, several adverse effects of alemtuzumab were described. Some of these were potentially dangerous. Infusion-associated reactions (IARs) were the most frequent, with a rate of incidence of $>90 \%$. Respiratory tract infections were even more common with alemtuzumab treatment. Secondary autoimmunity was also reported. Thyroid diseases, with percentages of 26\% in CAMMS223, $18 \%$ in CARE MS I and 17\% in CARE MS II, were the most frequent..$^{7-9}$ Idiopathic thrombocytopenic purpura (ITP) in $1 \%-3 \%$ of patients and anti-glomerular basement membrane (anti-GBM) disease (also called Goodpasture's syndrome) in $1 \%$ were observed. Other autoimmune adverse effects, such us neutropenia, hemolytic anemia, agranulocytosis and pancytopenia, were described in the Phase III clinical studies. ${ }^{8,9}$ Nevertheless, in Phase II and Phase III clinical studies, serious events were not significantly different among the treatment arms. ${ }^{7-9}$ In a 6-year extension study, the adverse effects of alemtuzumab were lower than those observed in CARE MS studies, whereas thyroid disease - with an initially higher incidence over 3 years - subsequently decreased. ${ }^{15}$

\section{IARs as AEs}

As seen in the other infused biologic drugs, IAR was the most frequent adverse event (AE) during treatment with alemtuzumab. In the Phase III clinical trials, IAR incidence was $>90 \%$, with the reactions being mild to moderate in severity. ${ }^{8,9}$ The reactions generally included headache, rash, pyrexia, nausea, flushing, urticaria, insomnia and pruritus. More than $10 \%$ of patients in the Phase III studies showed cardiac disorders, in particular tachycardia; ${ }^{17}$ therefore, physicians should be aware of the patients' condition in order to eventually treat these possible symptoms. It is thought that the IARs are due to allergic reactions of hypersensitivity mediated by immunoglobulin $\mathrm{E}$ and nonallergic cytokine release reactions induced by the recruitment of inflammatory cells or cell lysis. ${ }^{18-21}$ IARs decreased with each annual course of treatment and with subsequent infusions within a course. ${ }^{17}$ Severe IARs may be reduced by premedication with corticosteroids before and during infusion ${ }^{1}$ or by slowing or temporarily stopping the infusion. Incidence of IARs in clinical trials was higher in the group with alemtuzumab $24 \mathrm{mg}$ dose, but the reactions were of the same type in both the 12 and $24 \mathrm{mg}$ dose groups. ${ }^{8,9}$ All clinical trials reported severe IARs ranging from $1 \%$ to $3 \%$. One serious case of anaphylactic IAR occurred during the third treatment course, which was the first infusion in the extension study of CARE MS II. ${ }^{18}$ The patient presented swelling of the face, redness, as well as swelling and itching of the mouth and throat, along with coughing. The patient recovered after treatment with diphenhydramine, epinephrine and oxygen. ${ }^{17}$ Serious IARs have been also described in postmarketing reports: syncope, pulmonary infiltrates, acute respiratory distress syndrome, respiratory arrest, cardiac arrhythmias, myocardial infarction, acute cardiac insufficiency and cardiac arrest. ${ }^{1}$ Pretreatment procedures that mitigate IARs consist of corticosteroid administration immediately before each of the first 3 days of the infusion cycle and may also include antihistamines and/or antipyretics. ${ }^{2}$

\section{Recent cases of acute thrombocytopenia}

In a study by Ranganathan et al, ${ }^{22} 3$ cases of acute thrombocytopenia in 22 patients treated with alemtuzumab were observed. Soon after the first administration cycle, the 3 patients developed decrease in platelet count and bruising. These 3 acute and transient cases were the first to be described during alemtuzumab infusion. The reactions are different from those of ITP. ITP was reported as an adverse effect occurring months after alemtuzumab infusion with an immune-mediated mechanism. In addition, all 22 patients in the study had an asymptomatic platelet count decrease for 2 months, which later normalized. ${ }^{22}$ Decrease in platelet count also occurred in Phase III clinical studies, but these data were not published. This potential adverse effect of alemtuzumab should be monitored through postmarketing surveillance.

\section{Infections}

Infections were more frequent with alemtuzumab use and mainly involved infections of the upper and lower respiratory tract and the urinary tract. Infections were frequently caused by Herpes Simplex virus, Herpes Zoster virus and mycetes. Infections were seen in $66 \%$ of patients on alemtuzumab $12 \mathrm{mg}$ versus $47 \%$ of patients on IFN $\beta$ 1a in CAMMS223, in $67 \%$ versus $45 \%$, respectively, in CARE MS I ${ }^{8}$ and in $77 \%$ versus $66 \%$, respectively, in CARE MS II. ${ }^{9}$ Most infections were mild or moderate in severity. ${ }^{7-9}$ Serious infections were slightly higher with alemtuzumab than with IFN $\beta$ 1a in the pivotal studies. However, the overall incidence was low with alemtuzumab treatment compared to that 
with IFN $\beta$ 1a. In the CAMMS223 study, ${ }^{7}$ infections were $3 \%$ versus $2 \%$, respectively; in the CARE MS I study, ${ }^{8} 2 \%$ versus $1 \%$, respectively; and in CARE MS II, ${ }^{9} 4 \%$ versus $1 \%$, respectively. In the follow-up of the CAMMS223 study, the infection rate was $7 \%$ versus $3 \%$, respectively. Clinical trial extension studies showed that infections were higher during the first year and decreased subsequently ${ }^{12,23}$ (first year: $59.9 \%$, second year: $55.1 \%$, third year $48.2 \%$, and fourth year $46 \%$ ). Since herpes virus infections increased in the clinical trials with alemtuzumab, prophylactic treatment with an oral anti-herpes agent on the first day of alemtuzumab and for 1 month of each treatment cycle ${ }^{2}$ was introduced in the risk management plan. Moreover, before receiving treatment with alemtuzumab, patients who have not contracted chickenpox and who have not been vaccinated against Varicella zoster virus (VZV) should be tested for anti-VZV antibodies. Consequently, at least 6 weeks before treatment with alemtuzumab, patients should be vaccinated but should not receive live viral vaccines. ${ }^{2}$ In fact, the effects of live viral vaccines on the immune system after treatment with alemtuzumab are yet to be evaluated. Several cases of opportunistic infections, such as listeria meningitis, were reported. ${ }^{7,25}$ A single case of cerebral nocardiosis in a woman treated with alemtuzumab for RRMS was also observed. ${ }^{24}$ Recently, a case of pulmonary infection caused by a rare bacterium Nocardia $s p$., called Nocardia beijingesis, was reported in a woman after alemtuzumab treatment. The woman recovered after intravenous antibiotic therapy (meropenem). ${ }^{25}$ No cases of progressive multifocal leukoencephalopathy have been reported in RRMS patients treated with alemtuzumab. Deaths associated with infections in patients treated with alemtuzumab have not been reported. In open-label studies, the infections caused by immunosuppression are spirochetal gingivitis, pyogenic granuloma and listeria meningitis. The low incidence of opportunistic infections with alemtuzumab treatment could be explained by conservation of the innate immune system. ${ }^{26}$ It is probable that alemtuzumab depletes predominantly naïve $\mathrm{T}$ - and B-cells, whereas memory $\mathrm{T}$ - and B-cells are relatively unaffected. ${ }^{26-28}$ Infections were observed already with alemtuzumab use in leukemia. ${ }^{29}$ One case of listeria meningitis was reported in the CAMMS223 study ${ }^{7}$ and recently in 2 other cases. The 2 cases (Table 2) occurred after the first cycle of alemtuzumab treatment. ${ }^{30}$ In Case 1, a 47-year-old Caucasian female, with multiple sclerosis (MS) since 1992, alemtuzumab $12 \mathrm{mg}$ was given once a day for 5 days. The day following the fifth infusion, the patient developed cephalgia and subfebrile temperatures. On the third day, the fever rose to $40.1^{\circ} \mathrm{C}\left(104^{\circ} \mathrm{F}\right)$, and the MS symptoms worsened. Initially, ampicillin, ceftriaxone and aciclovir were administered. After positive cultures for Listeria monocytogenes in the cerebrospinal fluid (CSF), only ampicillin was continued for 21 days. Cranial MRI showed 2 new lesions and no signs of the infection. After antibiotic treatment, the patient rapidly improved. In Case 2, a 43-year-old Caucasian female with clinical signs of MS since 2014, treatment with alemtuzumab was introduced due to active disease. Alemtuzumab $12 \mathrm{mg}$ was administered once a day for 5 days. Three days after the last infusion, the patient developed subfebrile temperatures, and on the fourth day, the fever rose to $40.4^{\circ} \mathrm{C}\left(104.7^{\circ} \mathrm{F}\right)$. She was treated with cefuroxime but did not improve. As a consequence, she was admitted to the hospital, where ganciclovir and piperacillin/tazobactam were initiated. Two days after admission, the patient developed headache and meningism. After lumbar puncture, the patient was

Table 2 Summary of the 2 cases of listeria meningitis

\begin{tabular}{|c|c|c|}
\hline \multirow[t]{2}{*}{ Features } & \multirow{2}{*}{$\frac{\text { Case } \mathrm{I}^{30}}{\text { Alemtuzumab } 12 \mathrm{mg} \text { daily for } 5 \text { days }}$} & \multirow{2}{*}{$\begin{array}{l}\text { Case } 2^{30} \\
\text { Alemtuzumab } 12 \mathrm{mg} \text { daily for } 5 \text { days }\end{array}$} \\
\hline & & \\
\hline Patient information & 47-year-old Caucasian female, MS since 1992. & 43-year-old Caucasian female, MS since 2014. \\
\hline Time of onset & $\begin{array}{l}\text { The day following the fifth alemtuzumab } \\
\text { infusion, the patient developed cephalgia and } \\
\text { subfebrile temperatures. }\end{array}$ & $\begin{array}{l}\text { Three days after the last alemtuzumab infusion, the patient developed } \\
\text { subfebrile temperatures and on the fourth day, the fever rose to } 40.4^{\circ} \mathrm{C} \\
\left(104.7^{\circ} \mathrm{F}\right) . \text { Eight days after the last infusion, the patient developed headache } \\
\text { and meningism. }\end{array}$ \\
\hline Drug treatment & $\begin{array}{l}\text { During admission, ampicillin, ceftriaxone and } \\
\text { aciclovir were administered. After positive } \\
\text { cultures for Listeria monocytogenes, ampicillin } \\
\text { was continued for } 2 \text { I days. }\end{array}$ & $\begin{array}{l}\text { She was treated with cefuroxime but did not improve. During admission, } \\
\text { ganciclovir and piperacillin/tazobactam were initiated. After lumbar puncture, } \\
\text { the patient was treated with ampicillin, ceftriaxone and gentamicin. } \\
\text { After detection of L. monocytogenes, ceftriaxone was discontinued, while } \\
\text { ampicillin and gentamicin were continued for } 3 \text { weeks. }\end{array}$ \\
\hline Findings & At discharge, the patient had no sequelae. & $\begin{array}{l}\text { The patient recovered completely. After discharge, the patient was treated } \\
\text { with trimethoprim/cotrimoxazole for I week to prevent meningitis } \\
\text { recurrence. }\end{array}$ \\
\hline
\end{tabular}

Abbreviation: MS, multiple sclerosis. 
treated with ampicillin, ceftriaxone and gentamicin. After detection of L. monocytogenes, ceftriaxone was discontinued, while ampicillin and gentamicin were continued for 3 weeks. With this therapy, the patient improved. After discharge, the patient was treated with trimethoprim/cotrimoxazole for 1 week to prevent recurrence. Both cases of infection occurred after the infusions and may be correlated to immunosuppression induced by alemtuzumab. In order to reduce the risk of severe infections, dietary recommendations require exclusion of certain foods such as unpasteurized milk and raw meat, during and for 1 month after treatment with alemtuzumab. ${ }^{2}$ Given that signs of the infection may not be present initially, it is very important that immunosuppressed patients with normal neurological examinations are not overlooked. ${ }^{30}$ In clinical trials, there was an increased risk of infections with alemtuzumab treatment. ${ }^{7-9}$ Other organisms, such us human papillomavirus (HPV), caused uterine cervix infection in women treated with alemtuzumab (2\%). Consequently, annual screening for HPV is recommended for female patients. ${ }^{2}$ Recently, 2 cases of Cytomegalovirus infection have been reported with alemtuzumab treatment. The infections occurred after $\sim 1$ month from the first administration cycle (5 days). After pharmacological treatment, both patients recovered. ${ }^{31}$

\section{Autoimmune disease}

Autoimmune disease is probably the most relevant risk in treatment with alemtuzumab. It is hypothesized that development of autoimmunity is related to the repopulation of lymphocytes. T-cell repopulation occurs through reconstitution of $\mathrm{T}$-cells in the thymus or proliferation of mature cells that have not been depleted. ${ }^{32}$ After treatment with alemtuzumab, secondary autoimmunity was not related with the number of alemtuzumab courses ${ }^{11}$ Clinical studies showed that the risk of development of autoimmune disease was higher 6-60 months from the end of the infusion cycle. ${ }^{8,9}$ Thyroid disease was the most frequent adverse effect of secondary autoimmunity in patients treated with alemtuzumab. Hyperthyroidism (Graves' disease), hypothyroidism and thyroiditis were reported. In the Phase III clinical studies, thyroid disorders were seen in $29.6 \%$ of patients treated with alemtuzumab. ${ }^{8,9}$ In the 5-year follow-up of CAMMS223, thyroid disorders were found in 39\% of patients treated with alemtuzumab $12 \mathrm{mg}$ and in $29 \%$ of patients treated with alemtuzumab $24 \mathrm{mg} .{ }^{12,33}$ For this reason, thyroid function tests are recommended before treatment, every 3 months during treatment and up to 48 months after the last infusion of alemtuzumab. ${ }^{1}$ Neutropenia, hemolytic anemia, pancytopenia and agranulocytosis were other autoimmune AEs in the Phase III clinical trials. ${ }^{8,9}$ ITP, with an overall incidence of $2 \%$ in clinical studies, was a less-frequent but still high-risk AE, with occurrence over a period of 1-48 months from the end of treatment. In the CAMMS223 trial, ${ }^{7}$ ITP was observed in 6 patients treated with alemtuzumab, one of whom died of intracranial hemorrhage due to ITP. ${ }^{7}$ Current recommendations require a complete blood count with differential count before treatment, during treatment and up to 48 months after the last infusion of alemtuzumab because ITP can be serious or fatal. ${ }^{2}$ It is not yet established whether preexisting autoimmune disorders can increase the risk of secondary autoimmunity. In clinical studies, the patients who developed thyroid events were admitted to retreatment with alemtuzumab and had no worsening of thyroid disease. ${ }^{2}$ Scientific evidence on this subject is still insufficient and necessitates further evaluation. Undoubtedly, patients with preexisting autoimmune disease require more caution and monitoring. A recent report described the development of severe thrombocytopenia and thyroid autoimmune disease in 2 patients after 9 and 11 months, respectively, from the first infusion cycle with alemtuzumab. These 2 patients had previously faced suspended fingolimod treatment due to severe lymphocytopenia. This suggests that the severe lymphocytopenia developed after treatment with DMT may be a risk factor for ITP with alemtuzumab. ${ }^{34}$ These risk factors require careful monitoring. Autoimmune nephropathy is less frequent. In clinical studies, only 4 cases $(0.3 \%)$ of glomerulonephritis were observed. Nephropathy, with increase of serum creatinine, hematuria and/or proteinuria, developed over 4-39 months after the end of the treatment. Urinalysis and monitoring of creatinine are recommended before starting treatment, during treatment and up to 48 months after the last infusion of alemtuzumab. ${ }^{35}$ Four cases of renal disease, including 2 cases of membranous glomerulonephritis (MGN) and 2 of anti-GBM disease, were reported. The patients with MGN showed improvement with diuretics and/or angiotensin-converting enzyme (ACE) inhibitors, while the 2 cases of antiGBM disease improved after treatment with plasmapheresis, cyclophosphamide and glucocorticosteroids. ${ }^{36}$ Meyer et al ${ }^{37}$ reported 2 cases of anti-GBM disease, which required renal transplantation. Since secondary autoimmunity is a frequent AE with delayed onset, patients should be alert to symptoms during treatment and be monitored in the long term. Serum level of interleukin 21 has been proposed as a biomarker to predict secondary autoimmunity in the long term after treatment with alemtuzumab, but detection tests have not 
proved useful until now. In fact, no markers are available to identify the risk of secondary autoimmunity in patients. ${ }^{32,38}$ Hopefully, further studies on biomarkers will help identify patients at high risk and low risk of secondary autoimmunity after alemtuzumab treatment. Early detection is critical in the treatment of serious AEs. Therefore, use of the mandatory risk management plan, which includes regular clinical evaluations and laboratory tests, is imperative.

\section{A case of disseminated necrotizing leukoencephalopathy (DNL)}

Metz et $\mathrm{al}^{39}$ reported a case of DNL in a 33-year-old female, with 14 years of active RRMS. Prior to treatment with alemtuzumab, the patient was treated with IFN, natalizumab and fingolimod. Alemtuzumab $12 \mathrm{mg} / \mathrm{d}$ was given for 5 consecutive days. After 8 months from this first cycle of alemtuzumab, the woman, during the stable phase of disease, was hospitalized for anemia. Anemia showed no improvement, and the patient developed respiratory infection and went into septic shock, dying 6 days after admission to the hospital. Brain autopsy showed inactive MS lesions and several recent necrotizing lesions, indicating that DNL developed with autoimmune hemolytic anemia and sepsis. ${ }^{39}$ This serious complication is of unknown etiology. It has been reported in HIV infections and in cases of leukemia and lymphoma ${ }^{40,41}$ during treatment with intrathecal or intravenous methotrexate at high dose in combination with radiotherapy. It has also been observed in 1 case with oral methotrexate at low dose. ${ }^{42}$ In some reports, septic shock has been associated with multifocal necrotizing leukoencephalopathy, probably induced by an exaggerated inflammatory reaction. ${ }^{43}$ In this case, DNL could be caused by immunosuppression or infusion, with consequent respiratory infection and sepsis. DNL can be difficult to diagnose because symptoms appear immediately after treatment or after many months. ${ }^{44}$ Postmarketing surveillance is extremely important even in these rare cases.

\section{Malignancy}

Malignancies have been observed in the clinical trials, but the incidence in patients treated with alemtuzumab has not been high. Lymphoproliferative malignancies, such as lymphoma, Castleman disease and non-Epstein Barr virus-associated Burkitt's lymphoma, were reported at a similar rate in the control arms. In the CAMMS223 study, 3 cases of cancer occurred after treatment with alemtuzumab: breast cancer, non-Epstein Barr virus-associated Burkitt's lymphoma and cervical cancer, with onset from 22 to 64 months after an annual infusion cycle. In the IFN $\beta$ 1a group, colon cancer was reported after 36 months. Regarding the risk of cancer with alemtuzumab treatment, these data $(2.8 \%$ versus $0.9 \%)$ are insufficient. ${ }^{7}$ In CARE MS I, ${ }^{8}$ cancers were $0.5 \%$ in the alemtuzumab $12 \mathrm{mg}$ group versus $0 \%$ in the IFN $\beta$ 1a group. In CARE MS II, ${ }^{9}$ malignancies were $0.6 \%$ in the alemtuzumab $12 \mathrm{mg}$ group versus $1.5 \%$ in the IFN $\beta$ 1a group. Overall, in all clinical studies, 29 patients out of 1,486 alemtuzumab treated-patients developed malignancies. Six of these were stage 1 thyroid papillary carcinoma with onset 10-41 months from the last infusion. ${ }^{45}$ Thyroid cancer was detected in a patient with a lymph node. ${ }^{46} \mathrm{Six}$ cases of basal cell carcinoma, 5 with breast cancer and 4 with melanoma, with an incidence $>1 \%$, were detected. In the extension study of Phase II trial, 1 patient died due to non-EBV-associated Burkitt's lymphoma. Another patient developed Castleman disease (a prelymphomatous state) and, after treatment with cyclophosphamide, doxorubicin, rituximab and prednisolone, was in remission. ${ }^{9,12}$ Moreover, 1 case of Castleman disease, $^{48} 1$ of thyroid cancer ${ }^{49}$ and 1 of melanoma ${ }^{50}$ have been described individually. In a long-term follow-up cohort study, 10 out of 100 patients had precancerous or cancerous lesions. ${ }^{47}$ Furthermore, 1 case of Castleman disease, ${ }^{48} 1$ of thyroid cancer ${ }^{49}$ and 1 of melanoma ${ }^{50}$ have been described individually. Although the risk of cervical cancer after depletion of T-lymphocytes exists, there are no long-term data for higher individual incidence with respect to the base population. In all clinical trials, neoplasms have not been statistically more frequent with alemtuzumab than the comparator IFN $\beta$ $1 \mathrm{a}$ and since the malignancies may occur after many years from treatment, postmarketing vigilance is crucial to better the cancer risks in long-term alemtuzumab treatment.

\section{Pregnancy}

Data relating to the effects of alemtuzumab in pregnancy are still limited. ${ }^{1}$ Alemtuzumab is able to cross the placenta and can be a risk to the fetus. Thyroid disease presents particular risks in pregnancy. Graves's disease from the mother was transiently transferred to the newborn through the placenta. ${ }^{1}$ Current recommendations require that women of potential childbearing age should use contraception during treatment with alemtuzumab and for up to 4 months after a cycle. ${ }^{2}$ Alemtuzumab can be used in pregnancy only if the possible benefit to the fetus is greater than the potential risk. $^{2}$ Achiron et al report no adverse results in an analysis on the clinical development program of alemtuzumab in patients with RRMS: of the total 1,486 patients in the studies, 179 pregnancies occurred in 131 patients. Moreover, of the completed pregnancies, 104 (66\%) had 
healthy babies. Furthermore, the incidences of spontaneous abortion $(36 ; 21 \%)$ and stillbirth $(1 ; 0.6 \%)^{51}$ are no different from those of the general population. The available data are still insufficient for determining the risks of alemtuzumab on the fetus. Therefore, further studies are essential and alemtuzumab is contraindicated in pregnancy.

\section{Conclusion}

Alemtuzumab is an important drug added to DMTs for the treatment of RRMS. During the entire clinical development program, alemtuzumab demonstrated higher efficacy in RRMS compared to IFN $\beta$ 1a, reducing the risk of relapse and SAD. In naïve patients and in patients with inadequate response to previous therapy, its efficacy in active disease has been demonstrated. Recent data have showed a sustained effect with 1 complete treatment cycle. Furthermore, alemtuzumab, when compared to the 3 agents approved for RRMS, namely, IFN $\beta$ 1a, fingolimod and natalizumab, showed an efficacy similar to that of natalizumab. An increased risk of infections and autoimmunity with alemtuzumab was seen in the clinical trials. Since several of these can be very serious, strict and rigorous monitoring is required. Because there are still no predictive tools, such as markers, to identify the risk of secondary autoimmunity in patients treated with alemtuzumab, the selection of candidate patients for treatment with alemtuzumab is crucial. The selection could be based on familiarity of autoimmune diseases. Furthermore, an electronic file for each individual patient may contain information on similar AEs with other drugs that cause autoimmune effects. In the selection of patients to be treated, it may be useful to dosage anti-GBM serum antibodies. Ultimately, the same assay may be useful during treatment with alemtuzumab.

\section{Disclosure}

The authors report no conflicts of interest in this work.

\section{References}

1. US FDA. Lemtrada: Prescribing Information. Available from: http:// www.accessdata.fda.gov/drugsatfda_docs/label/2014/103948s51391bl. pdf. Accessed June 16, 2017.

2. EMA. Summary of Product of Characteristics. Available from: http://www.ema.europa.eu/docs/en_GB/document_library/EPAR_ Product_Information/human/003718/WC500150521.pdf. Accessed June 16, 2017.

3. Coles AJ, Wing MG, Molyneux P, et al. Monoclonal antibody treatment exposes three mechanisms underlying the clinical course of multiple sclerosis. Ann Neurol. 1999;46(3):296-304.

4. Moreau T, Thorpe J, Miller D, et al. Preliminary evidence from magnetic resonance imaging for reduction in disease activity after lymphocyte depletion in multiple sclerosis. Lancet. 1994;344(8918):298-301.
5. Coles A, Deans J, Compston A. Campath-1H treatment of multiple sclerosis: lessons from the bedside for the bench. Clin Neurol Neurosurg. 2004;106(3):270-274.

6. Hirst CL, Pace A, Pickersgill TP, et al. Campath 1-H treatment in patients with aggressive relapsing remitting multiple sclerosis. $J$ Neurol. 2008;255(2):231-238.

7. Investigators CT, Coles AJ, Compston DA, et al. Alemtuzumab vs. interferon beta-1a in early multiple sclerosis. $N$ Engl J Med. 2008; 359(17):1786-1801.

8. Cohen JA, Coles AJ, Arnold DL, et al. Alemtuzumab versus interferon beta $1 \mathrm{a}$ as first-line treatment for patients with relapsing-remitting multiple sclerosis: a randomised controlled phase 3 trial. Lancet. 2012;380(9856):1819-1828.

9. Coles AJ, Twyman CL, Arnold DL, et al. Alemtuzumab for patients with relapsing multiple sclerosis after disease-modifying therapy: a randomised controlled phase 3 trial. Lancet. 2012;380(9856): 1829-1839.

10. Giovannoni G, Cohen JA, Coles AJ, et al. Alemtuzumab improves preexisting disability in active relapsing-remitting MS patients. Neurology. 2016;87(19):1985-1992.

11. Tuohy O, Costelloe L, Hill Cawthorne G, et al. Alemtuzumab treatment of multiple sclerosis: long-term safety and efficacy. J Neurol Neurosurg Psychiatry. 2015;86(2):208-215.

12. Coles AJ, Fox E, Vladic A, et al. Alemtuzumab more effective than interferon beta-1a at 5-year follow-up of CAMMS223 clinical trial. Neurology. 2012;78(14):1069-1078.

13. Arnold D, Traboulsee A, Coles A, et al. Durable Effect of Alemtuzumab on MRI Activity in Treatment-Naive Active Relapsing-Remitting Multiple Sclerosis Patients: 4-Year Follow-up of CARE-MS I. Neurology. Presented at the AAN Meeting 2015 in Washington. Neurology. 2015.

14. Traboulsee A, Coles A, Cohen J, et al. Durable Effect of Alemtuzumab on MRI Outcomes in Patients With Relapsing-Remitting Multiple Sclerosis Who Relapsed on Prior Therapy: 4-Year Follow-up of CARE-MS II. Neurology. 2015;84(14 Supplement). Presented at the AAN Meeting 2015 in Washington. 2015.

15. La Ganke C HB, Berkovich R, et al. Durable effect of alemtuzumab on disability improvement in patients with relapsing-remitting multiple sclerosis who relapsed on a prior therapy. Neurology. Presented at the AAN Meeting 2015 in Washington. Neurology. 2015.

16. Kalincik T, Brown JW, Robertson N, et al. Treatment effectiveness of alemtuzumab compared with natalizumab, fingolimod, and interferon beta in relapsing-remitting multiple sclerosis: a cohort study. Lancet Neurol. 2017;16(4):271-281.

17. Caon C, Namey M, Meyer C, et al. Prevention and management of infusion-associated reactions in the comparison of alemtuzumab and Rebif® efficacy in multiple sclerosis (CARE-MS) program. Int J MS Care. 2015;17(4):191-198.

18. Genzyme Therapeutics. LEMTRADA Summary of Product Characteristics. Oxford: Genzyme Therapeutics Ltd; 2013.

19. Breslin S. Cytokine-release syndrome: overview and nursing implications. Clin J Oncol Nurs. 2007;11(1 suppl):37-42.

20. Maggi E, Vultaggio A, Matucci A. Acute infusion reactions induced by monoclonal antibody therapy. Expert Rev Clin Immunol. 2011;7(1): 55-63.

21. Namey M, Halper J, O’Leary S, Beavin J, Bishop C. Best practices in multiple sclerosis: infusion reactions versus hypersensitivity associated with biologic therapies. J Infus Nurs. 2010;33(2):98-111.

22. Ranganathan U, Kaunzner U, Foster S, Vartanian T, Perumal JS. Immediate transient thrombocytopenia at the time of alemtuzumab infusion in multiple sclerosis. Mult Scler. 1352458517699876. Epub 2017 Mar 1.

23. Henson LJ, Arnold DL, Cohen JA, et al. Incidence of infection decreases over time in alemtuzumab-treated patients with relapsingremitting multiple sclerosis: 4-year follow-up of the CARE-MS studies. Proceedings of the AAN Meeting; April 18-25, 2015; Washington, DC, USA. 
24. Penkert H, Delbridge C, Wantia N, Wiestler B, Korn T. Fulminant central nervous system nocardiosis in a patient treated with alemtuzumab for relapsing-remitting multiple sclerosis. JAMA Neurol. 2016; 73(6):757-759.

25. Sheikh-Taha M, Corman LC. Pulmonary Nocardia beijingensis infection associated with the use of alemtuzumab in a patient with multiple sclerosis. Mult Scler. 2017;23(6):872-874.

26. Havrdova E, Horakova D, Kovarova I. Alemtuzumab in the treatment of multiple sclerosis: key clinical trial results and considerations for use. Ther Adv Neurol Disord. 2015;8(1):31-45.

27. Hale G, Bright S, Chumbley G, et al. Removal of T cells from bone marrow for transplantation: a monoclonal antilymphocyte antibody that fixes human complement. Blood. 1983;62(4):873-882.

28. Jacobs P, Wood L, Fullard L, Waldmann H, Hale G. T cell depletion by exposure to Campath-1G in vitro prevents graft-versus-host disease. Bone Marrow Transplant. 1994;13(6):763-769.

29. Lin TS, Donohue KA, Byrd JC, et al. Consolidation therapy with subcutaneous alemtuzumab after fludarabine and rituximab induction therapy for previously untreated chronic lymphocytic leukemia: final analysis of CALGB 10101. J Clin Oncol. 2010;28(29): 4500-4506.

30. Rau D, Lang M, Harth A, et al. Listeria meningitis complicating alemtuzumab treatment in multiple sclerosis - report of two cases. Int J Mol Sci. 2015;16(7):14669-14676

31. Clerico M, De Mercanti S, Artusi CA, Durelli L, Naismith RT. Active CMV infection in two patients with multiple sclerosis treated with alemtuzumab. Mult Scler. 2017;23(6):874-876.

32. Jones JL, Phuah CL, Cox AL, et al. IL-21 drives secondary autoimmunity in patients with multiple sclerosis, following therapeutic lymphocyte depletion with alemtuzumab (Campath-1H). J Clin Invest. 2009; 119(7):2052-2061.

33. Daniels GH, Vladic A, Brinar V, et al. Alemtuzumab-related thyroid dysfunction in a phase 2 trial of patients with relapsing-remitting multiple sclerosis. J Clin Endocrinol Metab. 2014;99(1):80-89.

34. Obermann M, Ruck T, Pfeuffer S, Baum J, Wiendl H, Meuth SG Simultaneous early-onset immune thrombocytopenia and autoimmune thyroid disease following alemtuzumab treatment in relapsing-remitting multiple sclerosis. Mult Scler. 2016;22(9):1235-1241.

35. LEMTRADA. LEMTRADA REMS Program Overview. Available from: https:/www.lemtradarems.com/Docs/Pdf/LEMTRADA_REMS_ Program_Overview.pdf. Accessed August 1, 2015.

36. Jones JL, Coles AJ. Mode of action and clinical studies with alemtuzumab. Exp Neurol. 2014;262 Pt A:37-43.

37. Meyer D, Coles A, Oyuela P, Purvis A, Margolin DH. Case report of antiglomerular basement membrane disease following alemtuzumab treatment of relapsing-remitting multiple sclerosis. Mult Scler. 2013;2(1):60-63.
38. Azzopardi L, Thompson SAJ, Harding KE, et al. Predicting autoimmunity after alemtuzumab treatment of multiple sclerosis. $J$ Neurol Neurosurg Psychiatry. 2014;85(7):795-798.

39. Metz I, Rieckmann P, Kallmann B-A, Bruck W. Disseminated necrotizing leukoencephalopathy eight months after alemtuzumab treatment for multiple sclerosis. Acta Neuropathol Commun. 2016;4(1):81.

40. Pande AR, Ando K, Ishikura R, et al. Disseminated necrotizing leukoencephalopathy following chemoradiation therapy for acute lymphoblastic leukemia. Radiat Med. 2006;24(7):515-519.

41. Sakamaki H, Onozawa Y, Yano Y, et al. Disseminated necrotizing leukoencephalopathy following irradiation and methotrexate therapy for central nervous system infiltration of leukemia and lymphoma. Radiat Med. 1993;11(4):146-153.

42. Raghavendra S, Nair MD, Chemmanam T, Krishnamoorthy T, Radhakrishnan VV, Kuruvilla A. Disseminated necrotizing leukoencephalopathy following low-dose oral methotrexate. Eur J Neurol. 2007; 14(3):309-314.

43. Sharshar T, Gray F, Poron F, Raphael JC, Gajdos P, Annane D. Multifocal necrotizing leukoencephalopathy in septic shock. Crit Care Med. 2002;30(10):2371-2375.

44. Perry A, Schmidt RE. Cancer therapy-associated CNS neuropathology: an update and review of the literature. Acta Neuropathol. 2006; 111(3):197-212.

45. Miller T, Habek M, Coles A, et al. Analysis of data from RRMS alemtuzumab-treated patients in the clinical program to evaluate incidence rates of malignancy. Presented at 2014 Joint Meeting of Americas Committee for Treatment and Research in Multiple Sclerosis and European Committee for Treatment and Research in Multiple Sclerosis; September 2014; Boston, MA.

46. Lecumberri B, Arnold DA, Cohen JA, et al. Detection of thyroid malignancies in alemtuzumab-treated patients in the multiple sclerosis clinical development program. In: European Committee for Treatment and Research in Multiple Sclerosis; 2015; Barcelona.

47. Willis MD, Robertson NP. Alemtuzumab for multiple sclerosis. Curr Neurol Neurosci Rep. 2016;16(9):84.

48. Coles AJ. Alemtuzumab treatment of multiple sclerosis. Semin Neurol. 2013;33(1):66-73.

49. Ibitoye R, Wilkins A. Thyroid papillary carcinoma after alemtuzumab therapy for MS. J Neurol. 2014;261(9):1828-1829.

50. Pace AA, Zajicek JP. Melanoma following treatment with alemtuzumab for multiple sclerosis. Eur J Neurol. 2009;16(4):e70-e71.

51. Achiron A, Chambers C, Fox EJ, et al. Pregnancy outcomes in patients with active RRMS who received alemtuzumab in the clinical development program. Multiple Sclerosis Journal. 2015;21:581-582.
Therapeutics and Clinical Risk Management

\section{Publish your work in this journal}

Therapeutics and Clinical Risk Management is an international, peerreviewed journal of clinical therapeutics and risk management, focusing on concise rapid reporting of clinical studies in all therapeutic areas outcomes, safety, and programs for the effective, safe, and sustained use of medicines. This journal is indexed on PubMed Central, CAS,

\section{Dovepress}

EMBase, Scopus and the Elsevier Bibliographic databases. The manuscript management system is completely online and includes a very quick and fair peer-review system, which is all easy to use. Visit http://www.dovepress.com/testimonials.php to read real quotes from published authors. 